\title{
CAUSALIDADE ENTRE AS PRINCIPAIS BOLSAS DE VALORES DO MUNDO
}

\section{CAUSALITY AMONG THE MAIN STOCK EXCHANGES IN THE WORLD}

\section{HIRON PEREIRA FARIAS} Mestre em Estatística e Experimentação Agropecuária pelo Departamento de Ciências Exatas da Universidade Federal de Lavras (Ufla). Professor do Instituto de Matemática e Estatística da Universidade Federal de Goiás (UFG). Caixa postal 131 - campus Samambaia - Goiânia - GO - Brasil - CEP 74001-970 E-mail: hironpf@hotmail.com

\section{THELMA SÁFADI}

Doutora em Estatística pelo Instituto de Matemática e Estatística da Universidade de São Paulo (USP).

Professora do Departamento de Ciências Exatas da Universidade Federal de Lavras (Ufla). Departamento de Ciências Exatas, caixa postal 3037 - Lavras - MG - Brasil - CEP 37200-000

E-mail: safadi@ufla.br 


\section{RESUMO}

O objetivo deste trabalho foi analisar os mercados dos países emergentes que fazem parte do Bric, com exceção da Índia, buscando mostrar como os mercados do Brasil, da Rússia e China se comportam entre si e em relação ao mercado dos Estados Unidos. Analisou-se também como alguns países desenvolvidos do grupo G8, Estados Unidos, Reino Unido e Japão, se comportam. Em cada análise, ajustou-se um modelo VAR e buscou-se verificar o grau de dependência dentro e entre cada grupo, utilizando teste de causalidade de Granger, critérios de seleção de modelos, função resposta a impulso e decomposição da variância do erro de previsão. Nas análises realizadas, os mercados brasileiro e americano mostraram forte influência sobre os demais mercados, e, na análise entre os grupos, consideraram-se o mercado dos Estados Unidos do grupo ERJ e todos os mercados emergentes do grupo BRC. O mercado americano mostrou forte influência sobre os outros mercados.

\section{PALAVRAS-CHAVE}

Mercados emergentes e desenvolvidos; Índices de bolsas de valores; Teste de causalidade de Granger; Função resposta a impulso; Decomposição da variância do erro de previsão.

\section{ABSTRACT}

This work aimed to analyze the market of the emerging countries that are part of the Bric with the exception of India, attempting to show how the markets in Brazil, Russia and China behave between each other and how they behave in relation to the US market. It was also analyzed how some developed countries of the G8 group, USA, UK and Japan behave. In each analysis, a VAR model was adjusted and it was attempted to verify the degree of dependence in and between each 
group, using the Granger causality test, model selection criteria, impulse response function and forecast error variance decompositions. In the performed analyses, the Brazilian and American markets showed strong influence over the other markets, and, in the group analysis, the US market of the ERJ group and all of the emerging markets of group BRC were considered. The American market showed strong influence over the other markets.

\section{KEYWORDS}

Emerging and developed markets; Stock exchanges indices; Granger causality test; Impulse response functions; Forecast error variance decompositions.

\section{INTRODUÇÃO}

O processo histórico denominado globalização é bem recente, datando de I989 e I99I, com o colapso do bloco socialista e o consequente fim da guerra fria. A globalização se consolidou com a abertura comercial e livre circulação de capitais e serviços em escala mundial. As disputas acirradas no âmbito do mercado global, entre empresas e países, favoreceram a formação de blocos econômicos numa "guerra" de mercado, em que os parceiros estabelecem relações econômicas privilegiadas. Em consequência da globalização financeira, inúmeras são as oportunidades de novos investimentos e de novos negócios. Segundo Lamounier e Nogueira (2007, p. 35), "[...] a intensificação da interação econômica entre os países, sobretudo na última década, vem proporcionando uma expansão das oportunidades". Tendo em vista as recentes quedas das restrições ao capital internacional que atualmente se movimenta com maior liberdade e o crescente processo de intensificação das relações de trocas comerciais e de serviços entre os diversos países, os investidores começaram a perceber a possibilidade de expandir seus negócios para além de suas fronteiras domésticas, ampliando o seu horizonte até mesmo para os mercados emergentes (LAMOUNIER; NOGUEIRA, 2007, p. 35).

O marco central deste trabalho foi analisar séries temporais de retorno de índices econômicos de algumas das principais bolsas de valores do mundo, divididas em dois blocos, utilizando-se de ferramentas estatísticas multivariadas. Buscou-se verificar as relações dentro e entre os mercados do Bric, composto por Brasil, Rússia e China, com exceção da Índia, denotado neste trabalho grupo BRC e alguns países do G8 como Estados Unidos, Reino Unido e 
Japão, denominado grupo ERJ. Este trabalho também verificou a relação entre os mercados do grupo BRC e o mercado dos Estados Unidos.

\section{REFERENCIAL TEÓRICO}

Para Lamounier e Nogueira (2007, p. 35),

[...] entender o funcionamento e as relações entre os mercados emergentes e capitalizados torna-se um instrumento necessário para que o investidor saiba como alocar de maneira mais eficiente os recursos de modo a minimizar suas perdas.

Muitos são os estudos que procuram analisar o grau de associação entre os mercados de diversos países. Nesse sentido, Lamounier e Nogueira (2007) analisaram os mercados do Brasil, Rússia, Índia, China, México, Estados Unidos, Reino Unido e Japão, entre 1995 e 2002 e entre 2003 e 2005 . No período de I995-2000, verificou-se que apenas o retorno do mercado emergente da Rússia sofreu grandes impactos ante os choques dos retornos dos outros mercados. Entre 2003 e 2005 , os mercados do Brasil e México responderam de forma significativa aos choques nos retornos de demais mercados.

Para Pereira, Costa Júnior e Dantas (2000), com a globalização dos mercados e a incorporação das informações econômicas instantâneas, os fenômenos são captados permanentemente pelos países, influenciando o comportamento dos países e sendo influenciado por eles. Nessa linha de raciocínio, Pereira, Costa Júnior e Dantas (2000) procuraram identificar e aferir a magnitude das oscilações e o grau de causalidade entre os mercados dos Estados Unidos, Japão, Brasil, México, Venezuela, Chile, Peru e Argentina no período de julho de I994 a novembro de I998. Verificaram-se o efeito em cadeia e a simultaneidade do comportamento das bolsas desses mercados.

\subsection{MERCADOS EMERGENTES E DESENVOLVIDOS}

A sigla Bric foi criada em 200I pelos analistas de mercado do Banco Golmam Sachs para fazer referência a quatro países: Brasil, Rússia, Índia e China. Esses países têm sido apontados, nos últimos cinco anos, como os prováveis candidatos a crescer de forma vigorosa, juntando-se ao clube dos países desenvolvidos nas próximas décadas (BRIC, 2008a).

O movimento que culminou com a criação do G8 tem origem na crise do petróleo de i973 e na recessão econômica mundial que ela causou. Integram o 
G8 a França, os Estados Unidos, o Reino Unido, a Alemanha, a Itália, o Japão, o Canadá e a Rússia. Enquanto os seis primeiros participam de todos os encontros desde I975, o Canadá juntou-se aos demais no ano seguinte. Já a Rússia foi formalmente admitida apenas em 2006, quando sediou a primeira reunião do G8 em seu território. O país, entretanto, já participava das conversas desde I994 e foi aos poucos sendo recebido pelos outros sete, como um reconhecimento pelo esforço em abandonar a antiga economia socialista e implantar reformas democráticas $(\mathrm{G} 8,2008)$.

\section{2 ÍNDICES DE BOLSAS DE VALORES}

A bolsa de valores é o mercado organizado onde são negociadas ações de empresas de capital aberto (públicas ou privadas) e outros instrumentos financeiros (ASSAF NETO, 200I).

As ações representam a menor fração do capital social de uma empresa, ou seja, são o resultado da divisão do capital social em partes iguais, sendo o capital social o investimento dos donos na empresa, isto é, o patrimônio da empresa. Esse dinheiro compra máquinas, paga funcionários etc. O capital social, assim, é a própria empresa (ASSAF NETO, 200I).

Os movimentos de uma bolsa de valores são captados por meio de indices, que englobam o valor médio em moeda corrente de determinado grupo de ações, consideradas mais representativas no movimento total do mercado, ou de empresas atuantes em determinados setores da economia.

Segundo Fontes (2006, p. 47),

[...] os índices de mercado servem como referência para análise do comportamento dos preços de determinada ação, as quais obedecem, em linha geral, às de mercado, que são fielmente retratadas pelos índices.

Para que um índice possa efetivamente ser utilizado como instrumento de avaliação de desempenho de um mercado ou de uma bolsa, deve ser composto por uma suposta carteira de ativos que representa de forma mais eficiente possível o comportamento do mercado (FONTES, 2006, p. 47). Conforme Fontes (2006, p. 47), o critério de seleção das carteiras dos índices faz que a performance dos diferentes índices não seja a mesma, ainda que eles sejam representativos da mesma bolsa. Esse critério de seleção e o tipo de metodologia utilizada para a formação das carteiras são, portanto, os fatores que levam à personalização dos índices e impõem a necessidade de constantes revisões das carteiras de 
índices, a fim de isolar os ativos que tenham frequência mínima nos pregões e números mínimos de negócios.

O indice Bovespa (Ibovespa) é o mais importante indicador do desempenho médio das cotações das ações negociadas na Bolsa de Valores de São Paulo. Trata-se da formação de uma suposta carteira de investimentos que, atualmente, é composta de 64 ações, retratando a movimentação dos principais papéis negociados na Bovespa, representando não só o comportamento médio dos preços, mas também o perfil das negociações - do mercado à vista - observadas nos pregões. Essas ações, em conjunto, representam $80 \%$ do volume transacionado nos doze meses anteriores à formação da carteira. Como critério adicional, exige-se que a ação apresente, no mínimo, $80 \%$ de presença nos pregões do período. Portanto, o critério de corte é a liquidez do papel. Para que sua representatividade se mantenha ao longo do tempo, a composição da carteira é reavaliada a cada quatro meses. Essa reavaliação é feita com base nos últimos doze meses, quando são verificadas alterações na participação de cada ação (BOLSA DE VALORES DE SÃO PAULO, 2008).

$\mathrm{O}$ indice FTSE-100 é calculado pela FTSE the index company e composto por um rol das cem ações mais representativas da Bolsa de Valores de Londres, visando detectar movimentos de alta ou baixa nas cotações (CAVALCANTE; MISUMI; RUDGE, 2005).

Um de seus principais índices é o Dow Jones Industrial Average (DJIA), que é o valor avaliado de trinta grandes ações industriais, cujos negócios passam pela Bolsa de Nova York.

O índice Nikkei-225, o mais tradicional do mercado de ações japonês, foi implantado em I 6 de maio de I949. O índice Nikkei refere-se às flutuações das cotações de uma carteira formada por 225 ações mais negociadas e de maior capitalização desse mercado.

O índice Hang Seng da Bolsa de Hong Kong é constituído das 33 ações mais representativas do mercado, uma espécie de termômetro do mercado de ações de Hong Kong (BRIC, 2008a).

O indice RTS, calculado pela primeira vez em $\mathrm{I}^{\circ}$ de setembro de 1995 , que se tornou o principal referencial para a indústria de seguros russa, é calculado tanto em rublo russo quanto em dólar americano e baseia-se nas cinquenta mais líquidas e capitalizadas ações da bolsa (BRIC, 2008a).

\subsection{ANÁLISE ESTATÍSTICA}

Um modelo autorregressivo vetorial - VAR(p) - oferece um meio de deixar que os dados, e não o pesquisador, determinem a estrutura dinâmica de um modelo (PINDYCK; RUBINFELD, 2004). 
Seja $\mathrm{X}_{\mathrm{t}}=\left(\mathrm{x}_{\mathrm{I}}, \mathrm{X}_{2 \mathrm{t}}, \ldots, \mathrm{x}_{\mathrm{nt}}\right)$, denotado por um vetor $(\mathrm{n} \times \mathrm{I})$ de variáveis de séries temporais estacionárias. Dizemos que $X_{t}$ segue um modelo $\operatorname{VAR}(p)$ se:

$$
\mathrm{X}_{t}=\Phi_{0}+\Phi_{1} \mathrm{X}_{t-1}+\Phi_{2} \mathrm{X}_{t-2}+\ldots+\Phi_{p} \mathrm{X}_{t-p}+\mathrm{a}_{t}
$$

em que $a_{t}$ é ruído branco com vetor de médias nulo e variância $\Sigma$, denotado por $a_{\mathrm{t}} \sim \mathrm{RB}(\mathrm{O}, \Sigma), \Phi_{\circ}=\left(\varnothing_{\mathrm{IO}}, \varnothing_{2 \circ}, \ldots, \varnothing_{n \circ}\right)$ é um vetor $(\mathrm{n} \times \mathrm{I})$ de constantes, e $\Phi_{k}$ são matrizes (n $\mathrm{n}$ ) constantes (MORETTIN, 2008).

Assim, depois de estimar um VAR(p), é importante ser capaz de caracterizar nitidamente sua estrutura dinâmica. As respostas a impulso fazem isso ao mostrar como um choque em qualquer das variáveis se filtra através do modelo, afetando todas as demais variáveis endógenas, e, eventualmente retroage sobre a própria variável (PINDYCK; RUBINFELD, 2004, p. 50I).

Todo processo VAR(p) estacionário tem uma representação de Wold da forma

$$
\mathrm{X}_{t}=\mu+a_{t}+\Psi_{1} a_{t-1}+\Psi_{2} a_{t-2}+\ldots
$$

em que $\Psi_{s}$ refere-se às matrizes de médias móveis de ordem $(\mathrm{n} \times \mathrm{n})$ determinadas recursivamente.

Uma vez que uma ordenação recursiva tenha sido estabelecida, a representação de Wold $\mathrm{X}_{t}$ baseada nos erros ortogonais $\eta_{t}$ é dada por:

$$
\mathrm{X}_{t}=\mu+\Theta_{0} \eta_{t}+\Theta_{\mathrm{I}} \eta_{t-1}+\Theta_{2} \eta_{t-2}+\ldots
$$

em que $\Theta_{\circ}=B^{-\mathrm{r}}$ é uma matriz triangular inferior. As respostas a impulso de choques ortogonais $\eta_{j}$ são

$$
\frac{\partial x_{i, t+s}}{\partial \eta_{j, t}}=\frac{\partial x_{i, t}}{\partial \eta_{j, t-s}}=\theta_{i j}^{s}, i, j=1,2, \ldots, n ; \mathrm{s}>0
$$

em que $\theta_{i j}^{s}$ é o i,j-ésimo elemento de $\theta^{s}$. Um gráfico de $\theta_{i j}^{s}$ contra s é chamado de função resposta a impulso ortogonal de $\mathrm{x}_{\mathrm{i}}$ com relação a $\eta_{j}$. Com $\mathrm{n}$ variáveis, há $\mathrm{n}^{2}$ possíveis funções de resposta a impulso. 
A função resposta a impulso e a decomposição variância do erro de previsão apresentam a mesma informação, embora graficamente tenham maneiras diferentes de ser apresentadas (DIEBOLD, 2004).

Segundo Zivot e Wang (2005), a decomposição de variância de erro de previsão responde à seguinte pergunta: Qual porcentagem (proporção) da variância do erro de previsão ao prever $\mathrm{X}_{\mathrm{T}+\mathrm{h}}$ é decorrente de choque estrutural $\eta_{\mathrm{j}}$ ? Usando choques ortogonais $\eta_{j}$, o vetor h-passos à frente, com coeficientes VAR conhecidos, pode ser expresso como:

$$
\mathrm{X}_{t+h}-\mathrm{X}_{t+h / T}=\sum_{s=0}^{h-1} \Theta_{s} \eta_{t+h-s}
$$

Para uma variável específica $X_{i, T+h}$, esse erro de previsão tem a seguinte forma:

$$
\mathrm{X}_{i, T+h}-\mathrm{X}_{T+h / T}=\sum_{s=0}^{h-1} \theta_{i 1}^{s} \eta_{1, t+h-s}+\ldots+\sum_{s=0}^{h-1} \theta_{i n}^{s} \eta_{n, t+h-s}
$$

Uma vez que os erros estruturais são ortogonais, a variância do erro de previsão h-passos à frente é:

$$
\operatorname{Var}\left(x_{i, T+h}-x_{i, T+h \mid T}\right)=\sigma_{n_{j}}^{2} \sum_{s=0}^{h-1}\left(\theta_{i 1}^{s}\right)^{2}+\ldots+\sigma_{n_{j}}^{2} \sum_{s=0}^{h-1}\left(\theta_{i n}^{s}\right)^{2}
$$

em que $\sigma_{\eta_{j}}^{2}=\operatorname{var}\left(\eta_{j t}\right)$.

A parte da $\operatorname{Var}\left(x_{i, T+h}-x_{i, T+h \mid T}\right)$ decorrente de choques $\eta_{j}$ é dada por:

$$
\operatorname{DVEP}_{i j}(h)=\frac{\sigma_{n_{j}}^{2} \sum_{s=0}^{h-1}\left(\theta_{i j}^{s}\right)^{2}}{\sigma_{n_{j}}^{2} \sum_{s=0}^{h-1}\left(\theta_{i 1}^{s}\right)^{2}+\mathrm{L}+\sigma_{n_{j}}^{2} \sum_{s=0}^{h-1}\left(\theta_{i n}^{s}\right)^{2}} i, j=1,2, \ldots, n
$$

Segundo Gujarati (2006, p. 559),

[...] embora a análise de regressão lide com a dependência de uma variável em relação a outras, isso não implica necessariamente causalidade. Em outras palavras, a existência de uma relação entre variáveis não prova causalidade nem direção de influência. 
Para sistemas, Granger define causalidade em termos de previsibilidade: a variável $\mathrm{X}$ causa $\mathrm{Y}$, com respeito a um dado universo de informação (que inclui $\mathrm{X}$ e Y ), se o presente de $\mathrm{Y}$ pode ser previsto mais eficientemente usando esse passado, toda e qualquer outra informação disponível (incluindo valores passados de Y) sendo usada em ambos os casos (MORETTIN, 2008).

$\mathrm{O}$ teste de causalidade de Granger pressupõe que a informação relevante para a previsão das respectivas variáveis, $X_{t}$ e $Y_{t}$, está contida unicamente nos dados da série temporal destas.

Para testar se X causa $\mathrm{Y}$, a hipótese nula é $\sum_{i=1}^{p} \beta_{i}=0$ "X não causa Y", com estimativas de duas regressões.

Estimamos uma regressão de $\mathrm{Y}$ em relação a valores defasados de $\mathrm{Y}$, bem como a valores defasados de $\mathrm{X}$ na regressão irrestrita, isto é,

$$
Y=\sum_{i=1}^{p} \alpha_{i} Y_{t-i}+\sum_{i=1}^{p} \beta_{i} X_{t-i}+a_{t}
$$

Em seguida, uma regressão de Y apenas em relação a valores defasados de Y, a regressão restrita, é dada por:

$$
Y=\sum_{i=1}^{p} \alpha_{i} Y_{t-i}+a_{t}
$$

Utiliza-se a soma de quadrados dos resíduos de cada regressão para calcular a estatística $\mathrm{F}$ e testar se o grupo de coeficientes $\beta_{\mathrm{I}}, \beta_{2}, \mathrm{~K}, \beta_{p}$ é significativamente diferente de zero.

Se o valor da estatística $\mathrm{F}$ for superior ao valor crítico de $\mathrm{F}(\mathrm{q}, \mathrm{N}-\mathrm{K})$ no nível de significância selecionado, rejeita-se a hipótese nula, e, nesse caso, "X causa Y".

Para Zivot e Wang (2005, p. 405), "[...] a ordem da defasagem para o modelo $\operatorname{VAR}(\mathrm{p})$ pode ser determinada, usando critérios de seleção do modelo". A abordagem geral é ajustar modelos $\operatorname{VAR}(\mathrm{p})$ com ordens $\mathrm{p}=\mathrm{O}, \mathrm{I}, 2, \ldots, \mathrm{p}_{\max }$ e escolher o valor de p que minimiza alguns critérios de seleção de modelo. Os critérios de seleção de modelo para modelos VAR(p) têm a a seguinte forma:

$$
I C(p)=\ln |\Sigma \mu(p)|+\mathrm{C}_{T} \varphi(n, p)
$$

em que $\Sigma(p)=T^{-1} \sum_{t=1}^{T} \varepsilon \varepsilon$ é a matriz de covariância residual, sem uma correção do número de graus de liberdade de um modelo VAR(p); CT é uma sequência indexada pelo tamanho da amostra T; e $\varphi(n, p)$, uma função de penalidade a qual penaliza grandes modelos VAR(p). Os três critérios de informação mais 
comuns são os de Akaike (AIC), Schwarz-Bayesiano (BIC) e Hannan-Quinn (HQ):

$$
\begin{aligned}
& A I C(k)=\ln |\Sigma \mu(p)|+\frac{2}{T} p n^{2}(\text { Akaike }) \\
& B I C(k)=\ln |\Sigma \mu(p)|+\frac{\ln T}{T} p n^{2}(\text { Schwarz }) \\
& H Q(k)=\ln |\Sigma \mu(p)|+\frac{\ln \ln T}{T} p n^{2}(\text { Hannan - Quinn })
\end{aligned}
$$

\section{PROCEDIMENTO METODOLÓGICO}

A partir dos dados originais, foram obtidas as séries de retornos.

Se $\mathrm{X}_{\mathrm{t}}$ é o valor do índice no instante $\mathrm{t}$, o log retorno ou retorno é dado por

$$
\mathrm{R}_{\mathrm{t}}=\Delta \ln \mathrm{X}_{\mathrm{t}}=\ln \left(\mathrm{X}_{\mathrm{t}}\right)-\ln \left(\mathrm{X}_{\mathrm{t}-\mathrm{I}}\right)
$$

Os dados utilizados referem-se aos principais índices de fechamentos diários das bolsas de valores do mundo que foram coletados no banco de dados dos sites PlaDin e RTS Stock Exchange. Nas datas em que as bolsas estiveram fechadas em razão de feriados nacionais ou por qualquer outro motivo, os índices utilizados, para o dia em questão, foram aqueles referentes ao último dia de negociação antes da paralisação. A amostra compreende o período de 4 de dezembro de 2006 a 7 de novembro de 2008 , constando de 509 observações em cada uma das séries. O Quadro I apresenta os índices a serem analisados, assim como a respectiva sigla. As séries de índices foram divididas em dois grupos denominados BRC e ERJ, em que as componentes de cada grupo são respectivamente (Ibov, RTS, HS) e (DJ, FTS, NIK). Como a Rússia pertence aos dois grupos, adotou-se como critério econômico o fato de ela ser uma economia emergente. Em razão disso, optou-se por incluí-la no grupo do BRC. 


\section{QUADRO I}

\section{MERCADOS ANALISADOS E BOLSAS COM} SEUS ÍNDICES E SUAS SIGLAS

\begin{tabular}{llll}
\hline PAÍS & BOLSA & ÍNDICE & SIGLA \\
\hline Brasil & São Paulo & Ibovespa & Ibov \\
\hline Rússia & Moscou & RTS Index & RTS \\
\hline China & Hong Kong & Hang Seng & HS \\
\hline Estados Unidos & Nova York & Dow Jones & DJ \\
\hline Japão & Tóquio & Nikkei-225 & NIK \\
\hline Reino Unido & Londres & FTS-100 & FTS \\
\hline
\end{tabular}

Fonte: Elaborada pelos autores.

A seguir será definido como as análises foram realizadas:

I. Na análise de cada grupo, foi estimado um modelo VAR(p), e, na construção do VAR, utilizou-se o teste de causalidade de Granger para ordenar as séries. Os critérios de Akaike, Schwarz e Hannan-Quinn foram utilizados para determinar a ordem $\mathrm{p}$ do modelo VAR.

2. Depois de ajustado o modelo VAR(p), analisaram-se os índices que compõem o sistema gerado, utilizando o teste de causalidade de Granger e os gráficos das funções de respostas a impulsos e da decomposição da variância do erro de previsão.

3. Na análise entre grupos, analisou-se apenas o índice DJ contra os índices do grupo BRC, utilizando os mesmos passos mencionados no primeiro e segundo itens. Nessa análise dinâmica, o índice DJ virá primeiro, pois buscaremos observar se DJ está interferindo no BRC.

\section{RESULTADOS E ANÁLISES}

\section{ANÁLISE DOS ÍNDICES ECONÔMICOS DOS PAÍSES DO GRUPO BRC}

A Figura I apresenta os gráficos das séries de índices Ibov, RTS e HS, e seus respectivos retornos (Ribov, RRTS e RHS). 
Em uma análise dos gráficos dos retornos na Figura I, observa-se que os índices de retornos se desenvolvem ao longo do tempo, ao redor de um valor constante zero, e apresentam uma forma de equilíbrio estável. Nesse caso, pode-se concluir que os retornos dos índices Ibovespa, RTS e Hang Seng são estacionários.

\section{FIGURA I}

GRÁFICOS DAS SÉRIES DE ÍNDICES IBOV, RTS E HS, E DOS RETORNOS RIBOV, RRTS E RHS PERÍODO DE

$$
\text { 4.12.2006 A 7.II.2008 }
$$

índice lbovespa

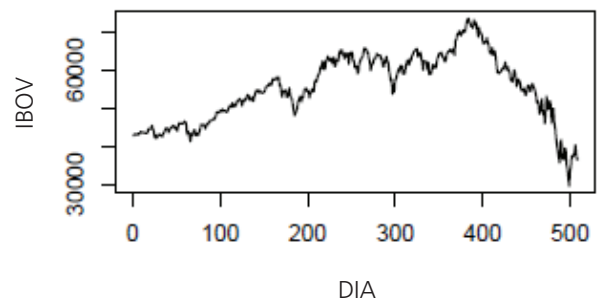

índice RTS

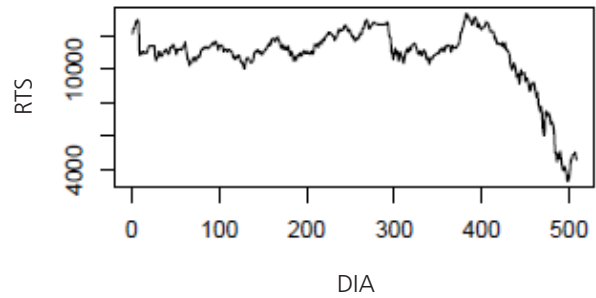

índice Hang Seng

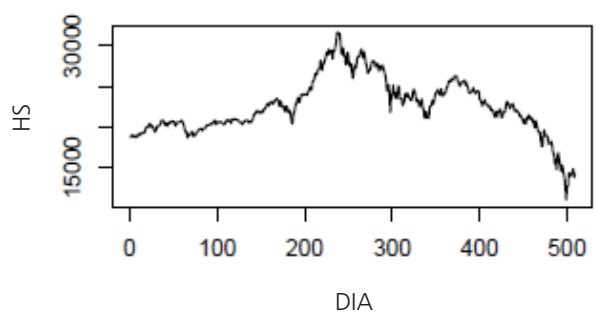

retorno Ibovespa

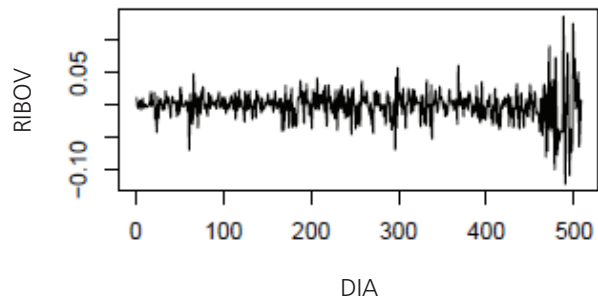

retorno RTS

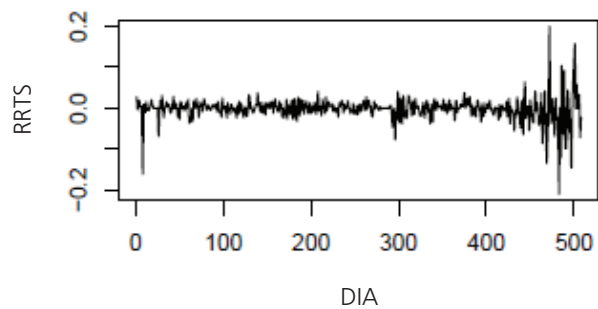

retorno Hang Seng

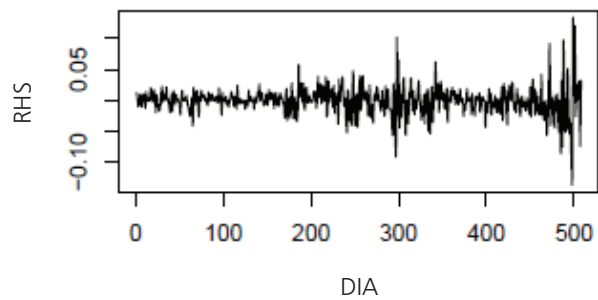

Fonte: Elaborada pelos autores. 
Na Tabela I, são apresentados os resultados do teste causalidade de Granger para os mercados do grupo BRC.

\section{TABELA I}

TESTE DE CAUSALIDADE DE GRANGER

PARA O GRUPO BRC

\begin{tabular}{lc}
\hline HIPÓTESE NULA & ESTATÍSTICA-F \\
\hline RRTS não causa Ribov & 0,05402 \\
\hline Ribov não causa RRTS & $72,5799^{*}$ \\
\hline RHS não causa Ribov & 1,03986 \\
\hline Ribov não causa RHS & $131,855^{*}$ \\
\hline RHS não causa RRTS & 0,33970 \\
\hline RRTS não causa RHS & $26,0922^{*}$ \\
\hline
\end{tabular}

*Teste significativo no nível de significância de Io\%.

Fonte: Elaborada pelos autores.

O índice Ribov causa no sentido de Granger os índices de retornos RRTS e RHS. No entanto, esses índices não causam, no mesmo sentido, o índice Ribov. Conforme resultados apresentados, a previsibilidade do retorno brasileiro não sofreu interferências dos mercados da Rússia e da China, mas ajuda a melhorar a previsibilidade dos retornos dos índices RTS e HS.

A previsibilidade do retorno do índice HS sofreu influência dos retornos das bolsas do Brasil e da Rússia, mas não influenciou na previsibilidade dos retornos de nenhum desses mercados.

Observa-se também que o índice de retorno RRTS causa, no sentido de Granger, o índice de retorno RHS. No entanto, o índice RHS não causa, no sentido de Granger, o índice RRTS. 


\section{TABELA 2}

CRITÉRIO DE SELEÇÃO DA ORDEM DA

DEFASAGEM PARA O GRUPO BRC

\begin{tabular}{cccc}
\hline DEFASAGEM & AIC & SC & HQ \\
\hline 0 & $-14,01113$ & $-13,98600$ & $-14,00127$ \\
\hline 1 & $-14,37832$ & $-14,27778^{*}$ & $-14,33888^{*}$ \\
\hline 2 & $-14,39814$ & $-14,22220$ & $-14,32913$ \\
\hline 3 & $-14,40206$ & $-14,15072$ & $-14,30347$ \\
\hline 4 & $-14,40582^{*}$ & $-14,07907$ & $-14,27765$ \\
\hline
\end{tabular}

*A ordem da defasagem selecionada pelos critérios.

$\mathrm{AIC}=$ critério de informação de Akaike; SC = critério de informação de Schwarz; HQ = critério de informação de Hannan-Quinn.

Fonte: Elaborada pelos autores.

Para ajustar um modelo VAR, o teste de causalidade de Granger sugere a seguinte ordenação: Ribov $\rightarrow$ RRTS $\rightarrow$ RHS. Os critérios de informação de Schwarz e Hannan-Quinn apresentados na Tabela 3 sugerem a ordem $\mathrm{p}=\mathrm{I}$ para o modelo.

Os gráficos das funções de respostas a impulsos e decomposição da variância do erro de previsão estão apresentados nas figuras 2 e 3, respectivamente.

A Figura 2 apresenta, nas colunas, as respostas ao impulso de Ribov, RRTS e RHS decorrente de um choque de uma unidade de desvio padrão em Ribov, RRTS e RHS, respectivamente.

Pode-se observar que, em razão de um choque unitário no Ribov, os retornos RRTS e RHS reagem positivamente. Em seguida, eles reagem negativamente para se estabilizarem em um terceiro momento. Em razão de um choque no índice RRTS, o índice Ribov mantém-se estável, e o RHS reage positivamente e se estabiliza no dia seguinte. Em razão do choque no índice de retorno RHS, os índices Ribov e RRTS reagem de forma negativa de modo não significativo. 


\section{FIGURA 2}

GRÁFICOS DAS FUNÇÕES RESPOSTAS A IMPULSOS DOS

RETORNOS DO GRUPO BRC DECORRENTES DE UM CHOQUE DE UM DESVIO PADRÃO NOS RETORNOS. NO EIXO DAS ABSCISSAS (X), TEM-SE O TEMPO EM DIAS, E, NO EIXO DAS ORDENADAS (Y), A MUDANÇA EM DESVIO PADRÃO
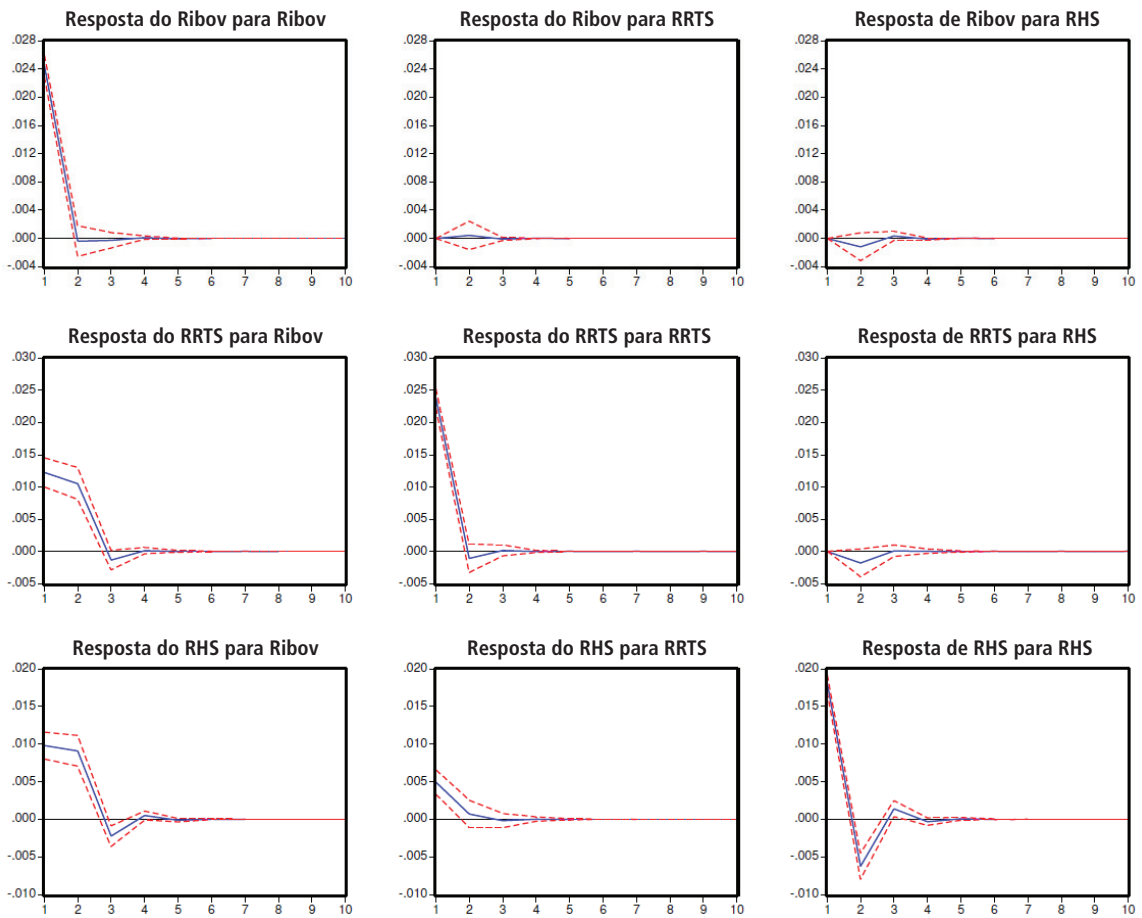

Fonte: Elaborada pelos autores.

A Figura 3, em colunas, representa as proporções da variância do erro de previsão de Ribov, RRTS e RHS em razão de um choque de uma unidade de desvio padrão em Ribov, RRTS e RHS, respectivamente.

Fazendo uma leitura na mesma ordem apresentada, pode-se observar que aproximadamente 100\%, 30\% e 30\% da variância do erro de previsão de Ribov, RRTS e RHS são decorrentes de um choque em Ribov. Em razão de um choque no retorno do índice RTS, são, respectivamente, zero, 70\% e 5\% as proporções das variâncias do erro de previsão de Ribov, RRTS e RHS, e, para um choque unitário em RHS, temos que $50 \%$ da proporção da variância do erro de previsão são decorrentes desse choque, e zero é a proporção da variância do erro de previsão dos retornos Ribov e RRTS decorrente desse choque. 
Observando a Figura 3 em linhas, temos o seguinte:

- Na primeira linha, conclui-se que, da variação total do erro de previsão do Ribov, IO०\% são decorrentes de um choque no Ribov, RRTS e RHS.

- Na segunda linha, conclui-se que, da variação total do erro de previsão do RRTS, 30\%, 70\% e ०\% são decorrentes de um choque no Ribov, RRTS e RHS.

- Na terceira linha, conclui-se que, da variação total do erro de previsão do RHS, 30\%, 5\% e 65\% são decorrentes de um choque, respectivamente, no Ribov, RRTS e RHS.

Conclui-se, das análises gráficas realizadas das funções respostas a impulsos e da decomposição da variância (figuras 2 e 3), que se trata de leituras equivalentes, isto é, há influência do mercado brasileiro sobre os mercados russo e chinês.

\section{FIGURA 3}

GRÁFICOS DA DECOMPOSIÇÃO DA VARIÂNCIA DO ERRO DE PREVISÃO DOS RETORNOS DO GRUPO BRC. NO EIXO DAS ORDENADAS (Y), TEM-SE A PORCENTAGEM DA VARIÂNCIA DE PREVISÃO DOS RETORNOS, E, NO EIXO DAS ABCISSAS (X),

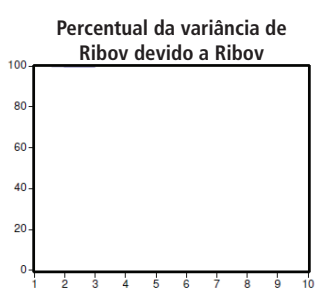

O HORIZONTE
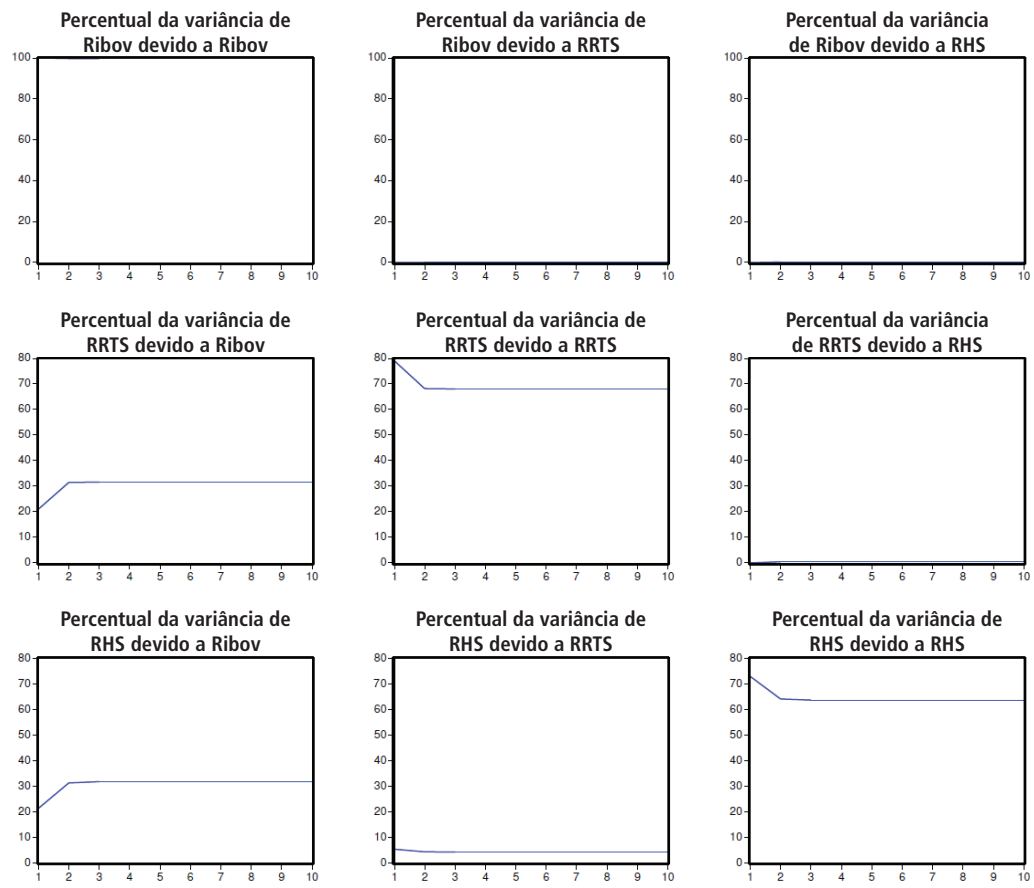

Fonte: Elaborada pelos autores. 


\subsection{ANÁLISE DOS ÍNDICES ECONÔMICOS DOS PAÍSES DO GRUPO ER J}

Nesta seção, serão apresentados os resultados da análise realizada com as séries de índices de retorno dos mercados dos Estados Unidos, do Reino Unido e do Japão, denotado por ERJ.

Em uma análise dos gráficos de retornos na Figura 4, pode-se concluir que os índices de retornos são estacionários, pois se desenvolvem ao longo do tempo ao redor do valor zero e apresentam uma variabilidade estável ao redor do valor constante zero.

\section{FIGURA 4}

GRÁFICOS DAS SÉRIES DE ÍNDICES DJ, FTS E NIK, E DOS RETORNOS RDJ, RFTS E RNIK NO PERÍODO DE

\section{I2.2006 A 7.II.2008}
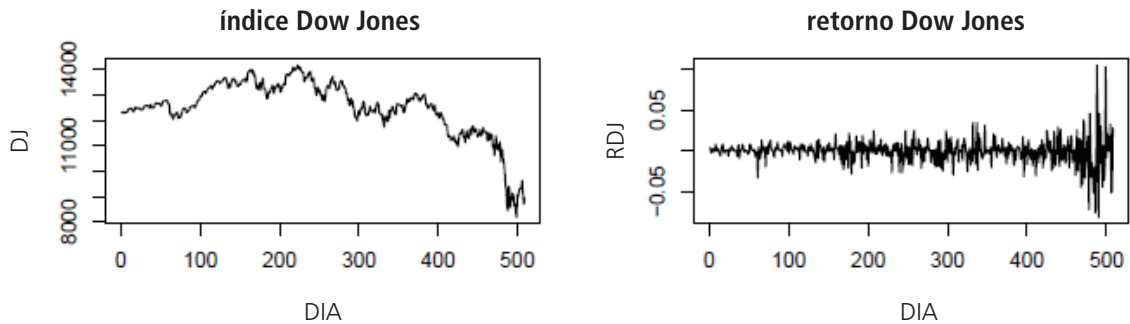

índice FTS
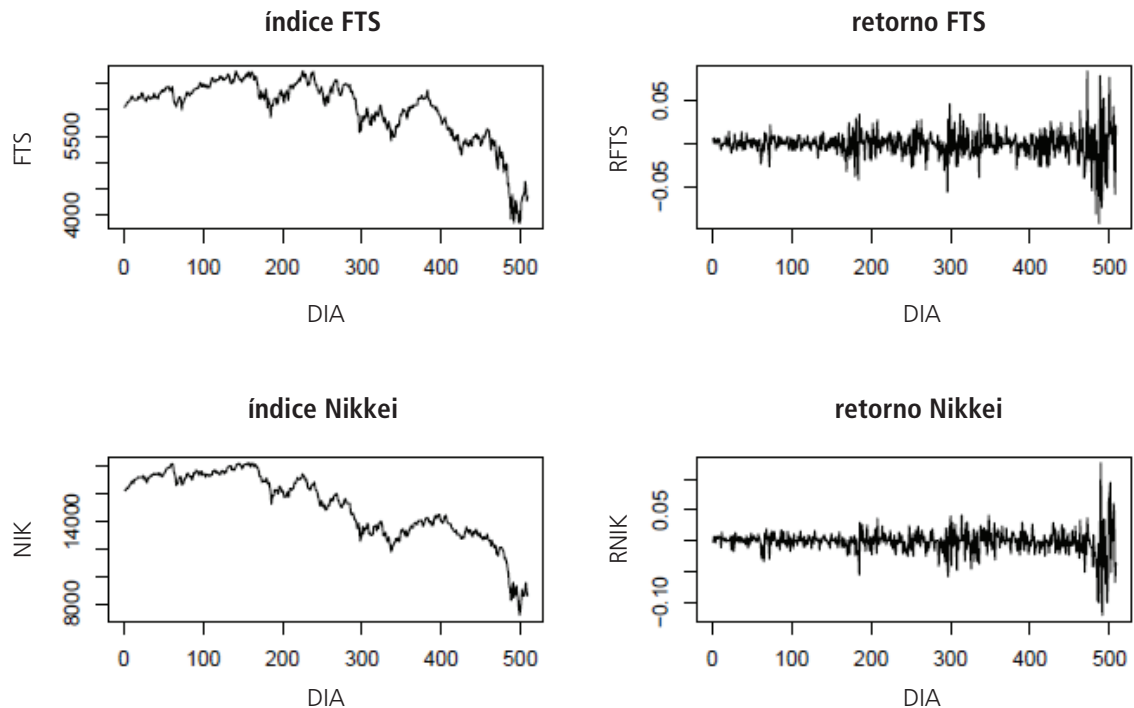

Fonte: Elaborada pelos autores. 


\section{TABELA 3}

CRITÉRIO DE SELEÇÃO DA ORDEM DA

DEFASAGEM PARA O GRUPO ERJ

\begin{tabular}{cccc}
\hline DEFASAGEM & AIC & SC & HQ \\
\hline 0 & $-16,3334$ & $-16,30831$ & $-16,32358$ \\
\hline 1 & $-17,24494$ & $-17,14440$ & $-17,20550$ \\
\hline 2 & $-17,34958$ & $-17,17364^{*}$ & $-17,28056$ \\
\hline 3 & $-17,42135^{*}$ & $-17,17001$ & $-17,32276^{\star}$ \\
\hline 4 & $-17,42124$ & $-17,09449$ & $-17,29307$ \\
\hline
\end{tabular}

*A ordem da defasagem selecionada pelos critérios.

AIC = critério de informação de Akaike; SC = critério de informação de Schwarz; HQ = critério de informação de Hannan-Quinn.

Fonte: Elaborada pelos autores.

Na Tabela 4, são mostrados os resultados do teste de causalidade de Granger para os mercados desenvolvidos dos Estados Unidos, do Reino Unido e do Japão. Observa-se que o índice RDJ causa, no sentido de Granger, os índices RFTS e RNIK, no entanto estes não causam, no sentido de Granger, o RDJ.

A previsibilidade do retorno americano RDJ não sofreu interferências dos mercados do Reino Unido e Japão, mas ajudou a melhorar significativamente a previsibilidade dos retornos dos índices RFTS e RNIK desses mercados.

A previsibilidade do retorno do índice NIK sofreu influência dos retornos das bolsas dos Estados Unidos e do Reino Unido, mas não influenciou a previsibilidade dos retornos de nenhum desses mercados.

\section{TABELA 4}

TESTE DE CAUSALIDADE DE GRANGER PARA O GRUPO ERJ

\begin{tabular}{lc}
\hline HIPÓTESE NULA & ESTATÍSTICA-F \\
\hline RRTS não causa Ribov & 1,73511 \\
Ribov não causa RRTS & $73,6876 *$ \\
\hline RHS não causa Ribov & 0,55953 \\
Ribov não causa RHS & $190,909 *$ \\
\hline RHS não causa RRTS & 2,27686 \\
RRTS não causa RHS & $71,0500 *$ \\
\hline
\end{tabular}

*Teste significativo no nível de significância de ı\%\%. Fonte: Elaborada pelos autores. 
Com base nos resultados observados do teste de causalidade de Granger (Tabela 5), a seguinte ordenação para estimar o modelo VAR é proposta: RDJ $\rightarrow$ RFTS $\rightarrow$ RNIK. De acordo com o critério de informação de Schwarz, na Tabela 4 , selecionou-se ordem $\mathrm{p}=2$ para ajustar o modelo VAR.

Ao ajustar um modelo VAR(2), os gráficos da função resposta a impulso e decomposição da variância estão plotados nas figuras 5 e 6 , respectivamente. $\mathrm{Na}$ Figura 5, apresentam-se, nas colunas, as respostas ao impulso de RDJ, RFTS e RNIK em razão de um choque de uma unidade de desvio padrão em RDJ, RFTS e RNIK, respectivamente.

Pode-se observar que, decorrente de um choque unitário no RDJ, a resposta de RDJ é instantaneamente positiva em torno de I, $5 \%$. Nos dois dias subsequentes, há reações negativas, chegando $-0,3 \%$ no terceiro dia, reagindo positivamente no quarto e quinto dias, e se estabilizando a partir do sexto dia.

\section{FIGURA 5}

GRÁFICOS DAS FUNÇÕES RESPOSTAS A IMPULSOS DOS RETORNOS DO GRUPO ERJ DECORRENTES DE UM CHOQUE DE UM DESVIO PADRÃO NOS RETORNOS. NO EIXO DAS ABSCISSAS (X), TEM-SE O TEMPO EM DIAS, E, NO EIXO DAS ORDENADAS (Y), A MUDANÇA EM DESVIO PADRÃO
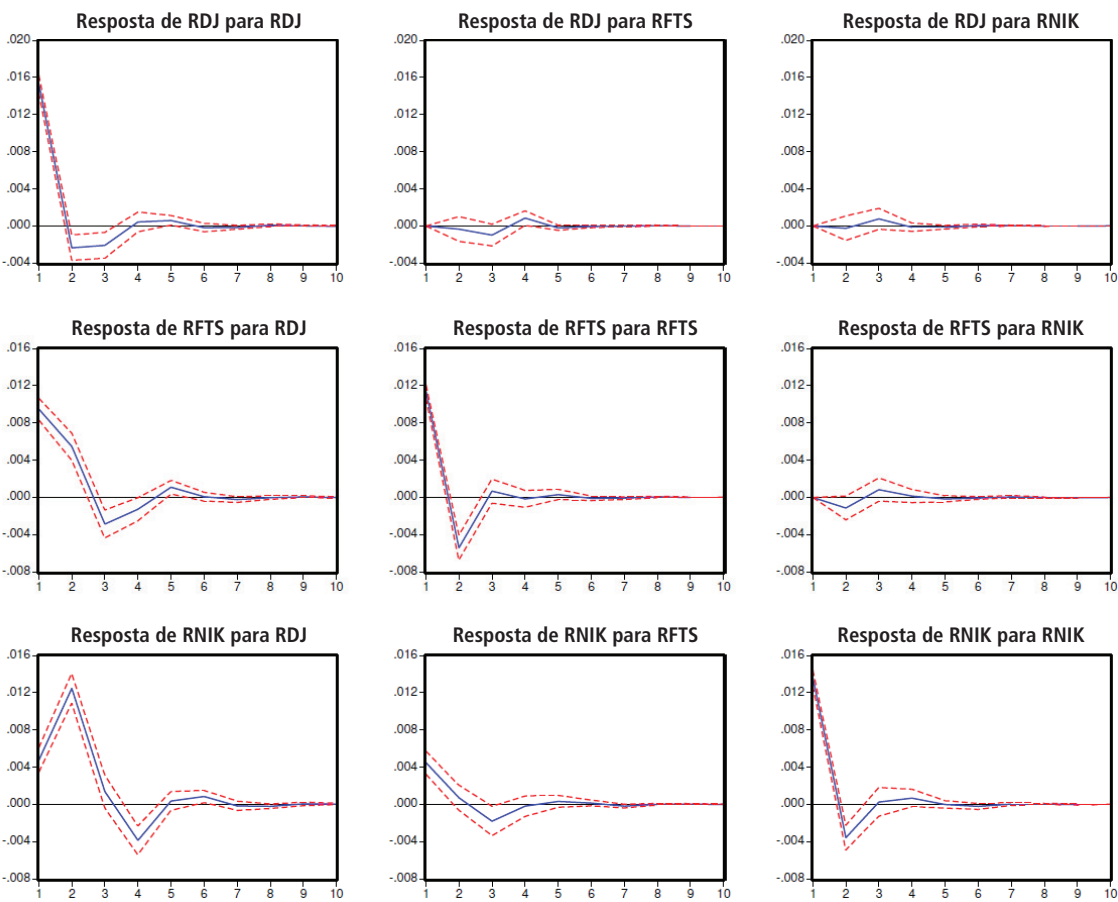

Fonte: Elaborada pelos autores. 
A resposta de RFTS é instantaneamente positiva em torno de $0,09 \%$. No segundo dia, reage positivamente, mas em queda, no terceiro dia, reage negativamente em torno de $-0,04 \%$, no quinto dia volta ser positiva e se estabiliza a partir do sexto dia. A resposta de RNIK é instantaneamente positiva em torno de I, $3 \%$. No segundo dia, continua a reagir positivamente, no terceiro reage negativamente em torno de $-0,04 \%$, e no quarto e quinto dias reage positivamente para estabilizar-se a partir do sexto dia. Em razão de um choque unitário no RFTS, a resposta de RFTS é instantaneamente positiva $0,4 \%$, no segundo dia reage negativamente, em torno de $-0,3 \%$, e se estabiliza a partir do terceiro dia. A resposta de RNIK reage instantânea e positivamente em torno de $0,4 \%$; no segundo dia reage positivamente, mas em queda, e no terceiro dia reage negativamente em torno de,, $02 \%$. O RDJ não reage de forma significativa. Os retornos reagem instantânea e positivamente no primeiro dia, reagem negativamente no segundo, voltam a reagir positivamente no terceiro e estabilizam-se a partir do quarto dia.

Na Figura 6, apresenta-se, nas colunas, a proporção da variância do erro de previsão de RDJ, RFTS e RNIK a um choque de uma unidade de desvio padrão no RDJ, RFTS e RNIK, respectivamente.

Na primeira coluna, observamos que aproximadamente I00\%, $45 \%$ e 40\% da variância do erro de previsão de RDJ, RFTS e RNIK, respectivamente, são decorrentes de um choque no RDJ.

Na segunda coluna, percebe-se que aproximadamente ०\%, 55\% e 5\% são, respectivamente, a proporção da variância do erro de previsão de RDJ, RFTS e RNIK decorrente de um choque em RFTS.

Na terceira coluna, aproximadamente ०\%, ०\% e 50\% são, respectivamente, a proporção da variância do erro de previsão de RDJ, RFTS e RNIK em razão de um choque em RNIK. 


\section{FigURA 6}

GRÁFICOS DA DECOMPOSIÇÃO DA VARIÂNCIA DO ERRO DE

PREVISÃO DOS RETORNOS DO GRUPO ERJ. NO EIXO DAS ORDENADAS (Y), TEM-SE A PORCENTAGEM DA VARIÂNCIA DE PREVISÃO DOS RETORNOS, E, NO EIXO DAS ABCISSAS (X),

\section{O HORIZONTE}
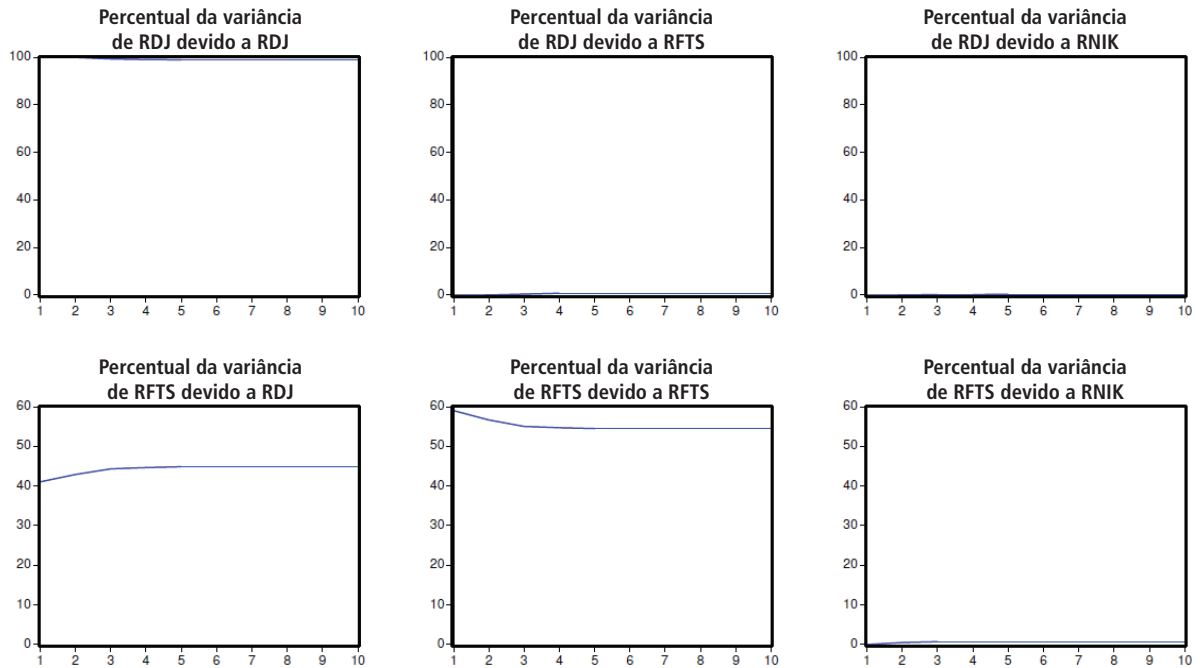

Percentual da variância de RNIK devido a RDJ
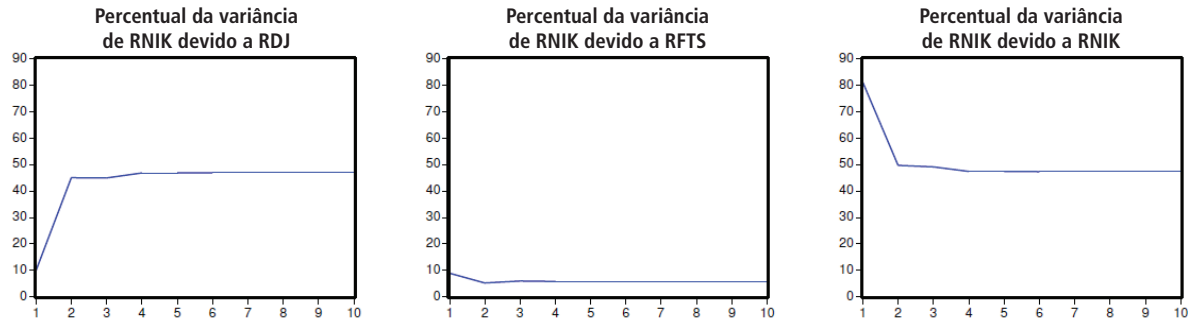

Fonte: Elaborada pelos autores.

Observando a Figura 6 em linhas, temos o seguinte:

- Na primeira linha, conclui-se que, da variação total do erro de previsão do RDJ, Io०\% são decorrentes de um choque no RDJ.

- Na segunda linha, conclui-se que, da variação total do erro de previsão do RFTS, $45 \%$, 55\% e o\% são decorrentes de um choque no RDJ, RFTS e RNIK.

- Na terceira linha, conclui-se que, da variação total do erro de previsão do RNIK, 45\%, $5 \%$ e 50\% são decorrentes de um choque, respectivamente, no RDJ, RFTS e RNIK. 
Na Figura 6, apresenta-se, na primeira linha, a variação total do erro de previsão do RDJ, e observa-se que Io०\% do erro de previsão é decorrente de um choque no RDJ; na segunda, tem-se a variação total do erro de previsão do RFTS, e observa-se que $45 \%$, $55 \%$ e o\% são decorrentes de um choque no RDJ, RFTS e RNIK, respectivamente; na terceira, temos a variação total do erro de previsão do RNIK, e observa-se que $45 \%$, $5 \%$ e $50 \%$ são decorrentes de um choque no RDJ, RFTS e RNIK, respectivamente.

Conclui-se, das análises gráficas realizadas, quando se observam as figuras 5 e 6, que trata-se de leituras equivalentes, isto é, há influência do mercado americano sobre os mercados inglês e japonês.

\subsection{ANÁLISE DOS ÍNDICES ECONÔMICOS DOS PAÍSES DO GRUPO G4}

Na Tabela 5, apresentam-se os resultados da causalidade de Granger para os mercados americano, brasileiro, russo e chinês, denotado por grupo $\mathrm{G}_{4}$.

\section{TABELA 5}

TESTE DE CAUSALIDADE DE GRANGER PARA O GRUPO G4

\begin{tabular}{lc}
\hline \multicolumn{1}{c}{ HIPÓTESE NULA } & ESTATÍSTICA-F \\
\hline Ribov não causa RDJ & 0,97611 \\
\hline RDJ não causa Ribov & $4,05968^{*}$ \\
\hline RRTS não causa RDJ & 0,10308 \\
\hline RDJ não causa RRTS & $62,2703^{*}$ \\
\hline RHS não causa RDJ & $7,15883^{*}$ \\
\hline RDJ não causa RHS & 99,4420 \\
\hline
\end{tabular}

*Teste significativo no nível de significância de ı०\%.

Fonte: Elaborada pelos autores.

O índice RDJ causa, no sentido de Granger, os índices Ribov, RRTS e RHS. Em outras palavras, o índice RDJ tem influência na previsão dos índices Ribov, RRTS e RHS, no entanto o índice RDJ não é influenciado de forma significativa na previsão por esses índices.

Os resultados do teste causalidade de Granger, comparando os índices Ribov, RRTS e RHS, são os mesmos da Tabela 2, por isso, não foram apresentados na Tabela 5 . 
Os resultados apresentados na Tabela 5 do teste de causalidade de Granger sugerem que a direção de causalidade seja RDJ $\rightarrow$ Ribov $\rightarrow$ RRTS $\rightarrow$ RHS, e pelo critério de informação de Schwarz, apresentado na Tabela 6, selecionou-se ordem $\mathrm{p}=\mathrm{I}$ para ajustar o modelo VAR.

\section{TABELA 6}

\section{CRITÉRIO DE SELEÇÃO DA ORDEM DA \\ DEFASAGEM PARA O GRUPO G 4}

\begin{tabular}{cccc}
\hline DEFASAGEM & AIC & SC & HQ \\
\hline 0 & $-20,34783$ & $-20,81431$ & $-20,33468$ \\
\hline 1 & $-20,78214$ & $-20,61457^{*}$ & $-20,71641$ \\
\hline 2 & $-20,83629$ & $-20,53468$ & $-20,71798^{*}$ \\
\hline 3 & $-20,84991^{*}$ & $-20,41425$ & $-20,67902$ \\
\hline 4 & $-20,83726$ & $-20,26755$ & $-20,61378$ \\
\hline
\end{tabular}

*A ordem da defasagem selecionada pelos critérios.

$\mathrm{AIC}=$ critério de informação de Akaike; SC = critério de informação de Schwarz; HQ = critério de informação de Hannan-Quinn.

Fonte: Elaborada pelos autores.

$\mathrm{Na}$ Figura 7, apresentam-se, na primeira coluna, as respostas ao impulso de RDJ, Ribov, RRTS e RHS, respectivamente, para um choque de uma unidade de desvio padrão em RDJ. Observa-se que Ribov, RRTS e RHS reagem instantânea e positivamente a um choque em RDJ; no segundo dia, Ribov reage positivamente mas em queda, e RDJ, RRTS e RHS reagem negativamente; ambos os índices estabilizam-se após o quarto dia.

Na Figura 8, apresentam-se, na primeira coluna, aproximadamente ioo\%, $55 \%, 20 \%$ e $20 \%$, respectivamente, da proporção da variância do erro de previsão de RDJ, Ribov, RRTS e RHS em razão do choque em RDJ. Na primeira linha, apresenta-se a variação total do erro de previsão do RDJ, e observa-se que aproximadamente ı००\% do erro de previsão é decorrente de um choque no próprio RDJ.

Conclui-se, das análises realizadas, quando se observam as figuras 7 e 8 , que trata-se de leituras equivalentes, isto é, há influência do mercado americano sobre os mercados emergentes brasileiro, russo e chinês. 


\section{FIGURA 7}

GRÁFICOS DAS FUNÇÕES RESPOSTAS A IMPULSOS DOS

RETORNOS DO GRUPO G4 DECORRENTES DE UM CHOQUE DE UM DESVIO PADRÃO NOS RETORNOS. NO EIXO DAS ABSCISSAS (X), TEM-SE O TEMPO EM DIAS, E, NO EIXO DAS ORDENADAS (Y), A MUDANÇA EM DESVIO PADRÃO

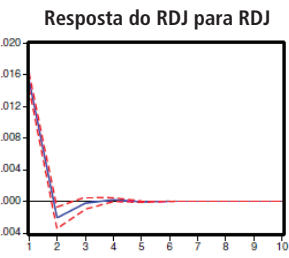

Resposta do RDJ para Ribov

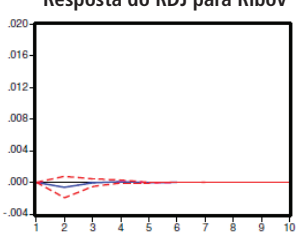

Resposta do Ribov para RDJ

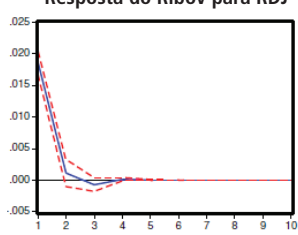

Resposta do RRTS para RDJ

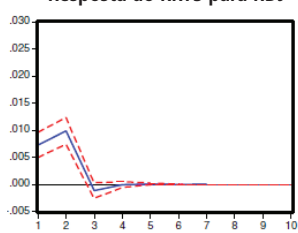

Resposta do RHS para RDJ

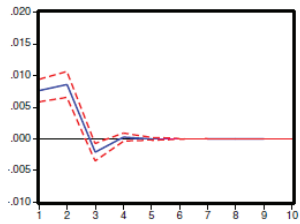

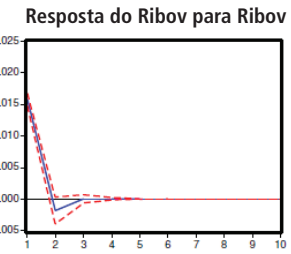

Resposta do RRTS para Ribov

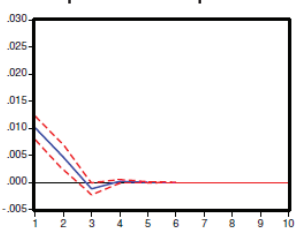

Resposta do RHS para Ribov

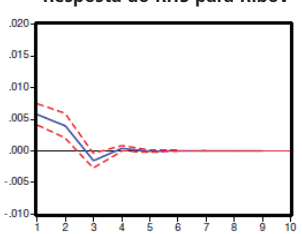

Resposta de RDJ para RRTS

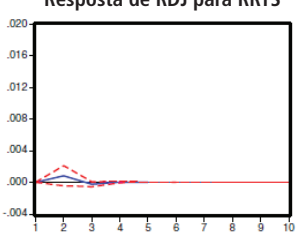

Resposta de Ribov para RRTS

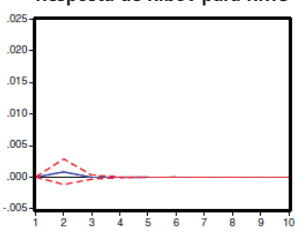

Resposta de RRTS para RRTS

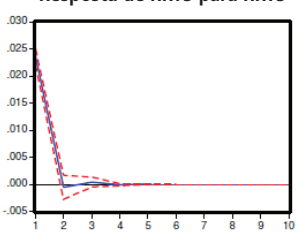

Resposta de RHS para RRTS

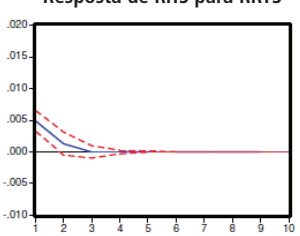

Resposta de RDJ para RHS

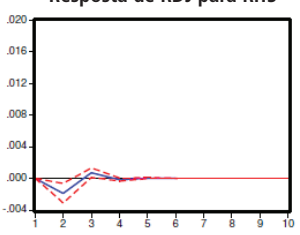

Resposta de Ribov para RHS

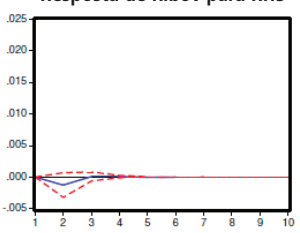

Resposta de RRTS para RHS

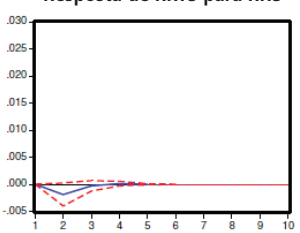

Resposta de RHS para RHS

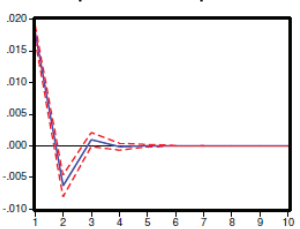

Fonte: Elaborada pelos autores. 


\section{FIGURA 8}

GRAFICOS DA DECOMPOSIÇAOO DA VARIANCIA DO ERRO

DE PREVISÃO DOS RETORNOS DO GRUPO G4. NO EIXO DAS

ORDENADAS (Y), TEM-SE A PORCENTAGEM DA VARIÂNCIA DE

PREVISÃO DOS RETORNOS, E, NO EIXO DAS ABCISSAS (X), O HORIZONTE DE TEMPO EM DIAS
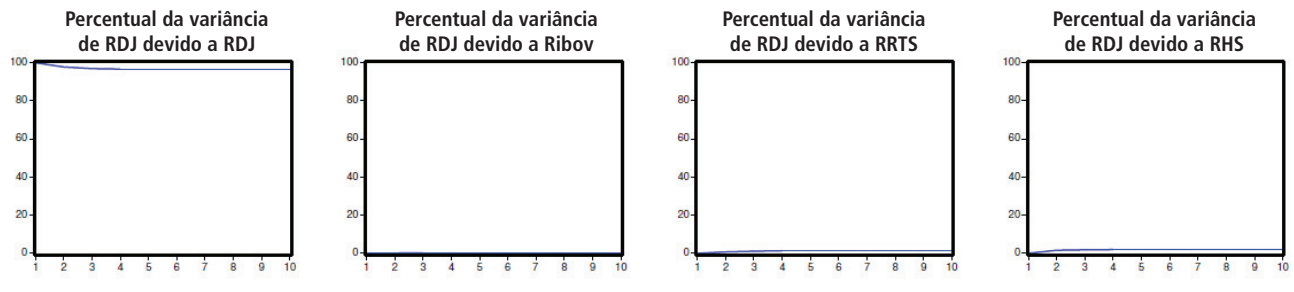

Percentual da variância

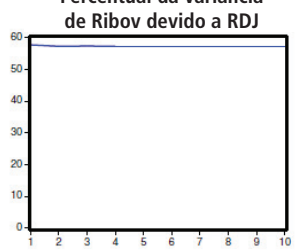

Percentual da variância

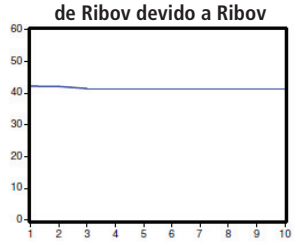

Percentual da variância

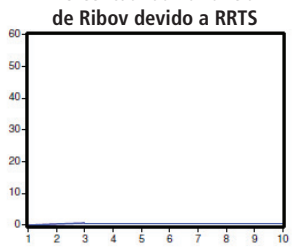

Percentual da variância

Percentual da variancia
de Ribov devido a RHS

Percentual da variância

Percentual da variância

Percentual da variância

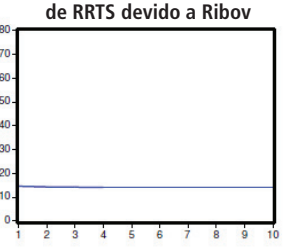

de RRTS devido a RRTS

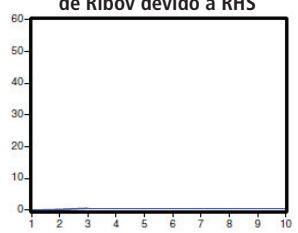

de RRTS devido a RDJ

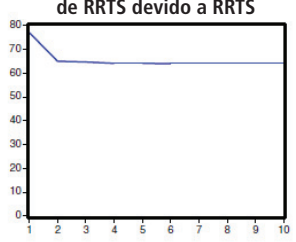

Percentual da variância

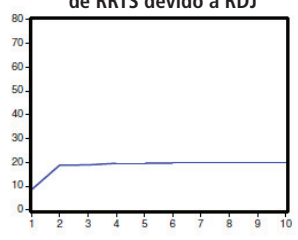

Percentual da variância

Percentual da variância

Percentual da variância

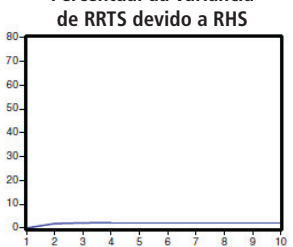

de RHS devido a RDJ

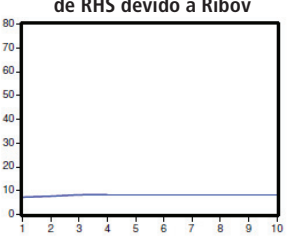

de RHS devido a RRTS

Percentual da variância

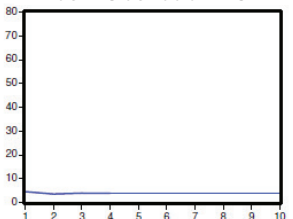

de RHS devido a RHS

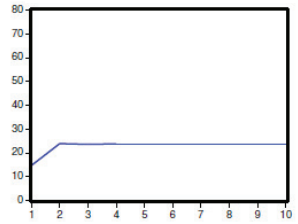

Fonte: Elaborada pelos autores.

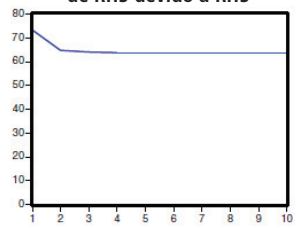

\section{CONCLUSÕES}

Por meio de modelo VAR, foram realizadas análises - por meio do teste de causalidade de Granger, da função resposta a impulso e da decomposição da variância do erro de previsão - das séries de retornos dos índices dos mercados emergentes brasileiro, russo e chinês, e dos mercados desenvolvidos: americano, inglês e japonês. 
O mercado brasileiro mostrou ter influência sobre os mercados russo e chinês, enquanto o mercado brasileiro não é influenciado por eles.

O mercado americano mostrou ter influência na previsão dos índices dos mercados inglês e japonês, enquanto o mercado americano não é influenciado por nenhum deles.

Para estimar os retornos dos índices dos mercados brasileiro, russo e chinês, é importante considerar o índice Dow Jones, pois esses mercados são influenciados pelo mercado americano.

\section{REFERÊNCIAS}

ASSAF NETO, A. Mercado financeiro. 4. ed. São Paulo: Atlas, 200I.

BOLSA DE VALORES DE SÃO PAULO. Disponível em: <http://www.bovespa.com.br>. Acesso em: 8 ago. 2008.

BOX, G. E. P.; JENKINS, G. M.; REINSEL, G. C. Time series analysis: forecasting and control. 3. ed. Englewood Cliffs: Prentice Hall, I994.

BRIC: Brasil, Rússia, Índia e China. Disponível em: <http://www2.camara.gov.br/fiquePorDentro/ temasanteriores/bric>. Acesso em: 9 ago. 2008a.

BRIC: Brasil, Rússia, Índia e China: o que é, características comuns dos países, economia, informações gerais, desenvolvimento econômico. Disponível em: <http://www.suapesquisa.com/ pesquisa/bric.htm>. Acesso em: 8 ago. 2008b.

BROCKWELL, P. J.; DAVIS, R. A. Introduction to time series and forecasting. 2. ed. New York: Springer, 2002.

CAVALCANTE, F.; MISUMI, J. F.; RUDGE, L. F. Mercado de capitais: o que é, como funciona. 6 ed. Rio de Janeiro: Campus, 2005.

CRYER, J. D.; CHAN, K. Times series analysis: with applications in R. 2. ed. New York: Springer, 2008.

DIEBOLD, F. X. Elements of forecasting. 3. ed. Pensylvania: Thomson, South-Western, 2004.

FONTES, R. E. Construção de um índice agrícola para o mercado derivativo de commodities agrícolas na BM\&F. 2006. I47 p. Tese (Doutorado em Administração)-Universidade Federal de Lavras, Minas Gerais, 2006.

G8. Disponível em: <http://www.brasilescola.com/geografia/g8.htm>. Acesso em: Io set. 2008. GUJARATI, D. Econometria básica. 4. ed. Rio de Janeiro: Campus, 2006.

LAMOUNIER, W. M.; NOGUEIRA, E. M. Causalidade entre os retornos de mercados de capitais emergentes e desenvolvidos. Revista de Contabilidade Finanças, São Paulo, v. ı8, n. 43, p. 34-48, jan./abr. 2007.

MORETTIN, P. A. Econometria financeira: um curso em séries temporais financeiras. São Paulo: E. Blucher, 2008 .

PEREIRA, A. F. O. A.; COSTA JÚNIOR, N. C. A.; DANTAS, A. B. Causalidade e co-integração das principais bolsas de valores do mundo e da América Latina. In: ENCONTRO DA ANPAD, 24., 2000, Florianópolis. Anais... Florianópolis: EnAnpad, 2000. Disponível em: <http://www.anpad. org.br/enanpad/2002/dwn/enanpad2002-fin-806.pdf.>. Acesso em: 2 ago. 2008. 
PEREIRA, A. F. O. A.; DANTAS, A. B; COSTA JÚNIOR, N. C. A. Estimação da co-integração das principais bolsas da América Latina, Estados Unidos e Japão pela metodologia Johanse. In: ENCONTRO DA ANPAD, 26., 2002, Salvador. Anais... Salvador: EnAnpad, 2002. Disponível em: <http://www.anpad.org.br/evento.php?acao=trabalho\&cod_edicao_subsecao=49\&cod_evento_ edicao=6\&cod_edicao_trabalho=2522>. Acesso em: 3 ago. 2008.

Crise, co-integração e previsão: uma aplicação nos mercados acionários da América Latina, Estados Unidos e Japão e Inglaterra. In: ENCONTRO DA ENANPAD, 28., 2004, Curitiba. Anais... Curitiba: EnAnpad, 2004. Disponível em: <http://www.anpad.org.br/evento.php?_=I\&acao=trabalho\&cod_edicao_subsecao=39\&cod_evento_edicao=8\&cod_edicao_ trabalho=II55\&idioma=3\#self $>$. Acesso em: 4 ago. 2008.

PINDYCK, R. S.; RUBINFELD, D. L. Econometria: modelos e previsões. 4. ed. Rio de Janeiro: Campus, 2004.

PLANETA Dinheiro. Disponível em: <http://www.pladin.uol.com.br>. Acesso em: I ago. 2008. RTS - Rússia Stock Exchange. Disponível em: <http://www.rts.ru/?tid=54I >. Acesso em: ${ }^{\circ}$ dez. 2008.

SILVA, W. S. Modelagem da volatilidade dos índices financeiros Ibovespa, Dow Jones e Standard \& Poors utilizando modelos da classe ARCH. 2003. 95 p. Dissertação (Mestrado em Estatística e Experimentação Agropecuária)-Universidade Federal de Lavras, Minas Gerais, 2003.

ZIVOT, E.; WANG, J. Modeling financial time series with S-plus. 2. ed. New York: Springer, 2005. 